\title{
Isolation and characterization of $T$ cells from human prenatal skin
}

Reitermaier R. ${ }^{1}$, Ayub T. ${ }^{1}$, Kienzl P. ${ }^{1}$, Scharrer A. ${ }^{2}$, Mildner M. ${ }^{3}$, Worda C.4 , Eppel W.4, Schuster C. ${ }^{1}$, Elbe-Bürger A. ${ }^{1}$

'Department of Dermatology, Division of Immunology, Allergy and Infectious Diseases, Medical University of Vienna, Austria 2Department of Pathology, Medical University of Vienna, Austria

3Department of Dermatology, Research Division of Biology and Pathobiology of the Skin, Medical University of Vienna, Vienna, Austria ${ }^{4}$ Department of Obstetrics \& Gynecology, Medical University of Vienna, Austria

\section{Introduction \& Study Aim}

Adult human skin is home to huge numbers of T cells that as pivotal part of the immune system fulfill important roles in immune surveillance and play a key role in cutaneous immunity. T cells are already present in fetal skin, yet their function remains elusive.

The aim of this study is to delineate the nature and significance of fetal skin $T$ cells and to compare it with T cells in adult skin. As T cells are extremely rare in fetal skin we compared established and novel isolation techniques and succeeded to enrich them in sufficient numbers to better characterize them.

\section{Methodology}

Sample preparation Single cell suspension _ FACS analysis

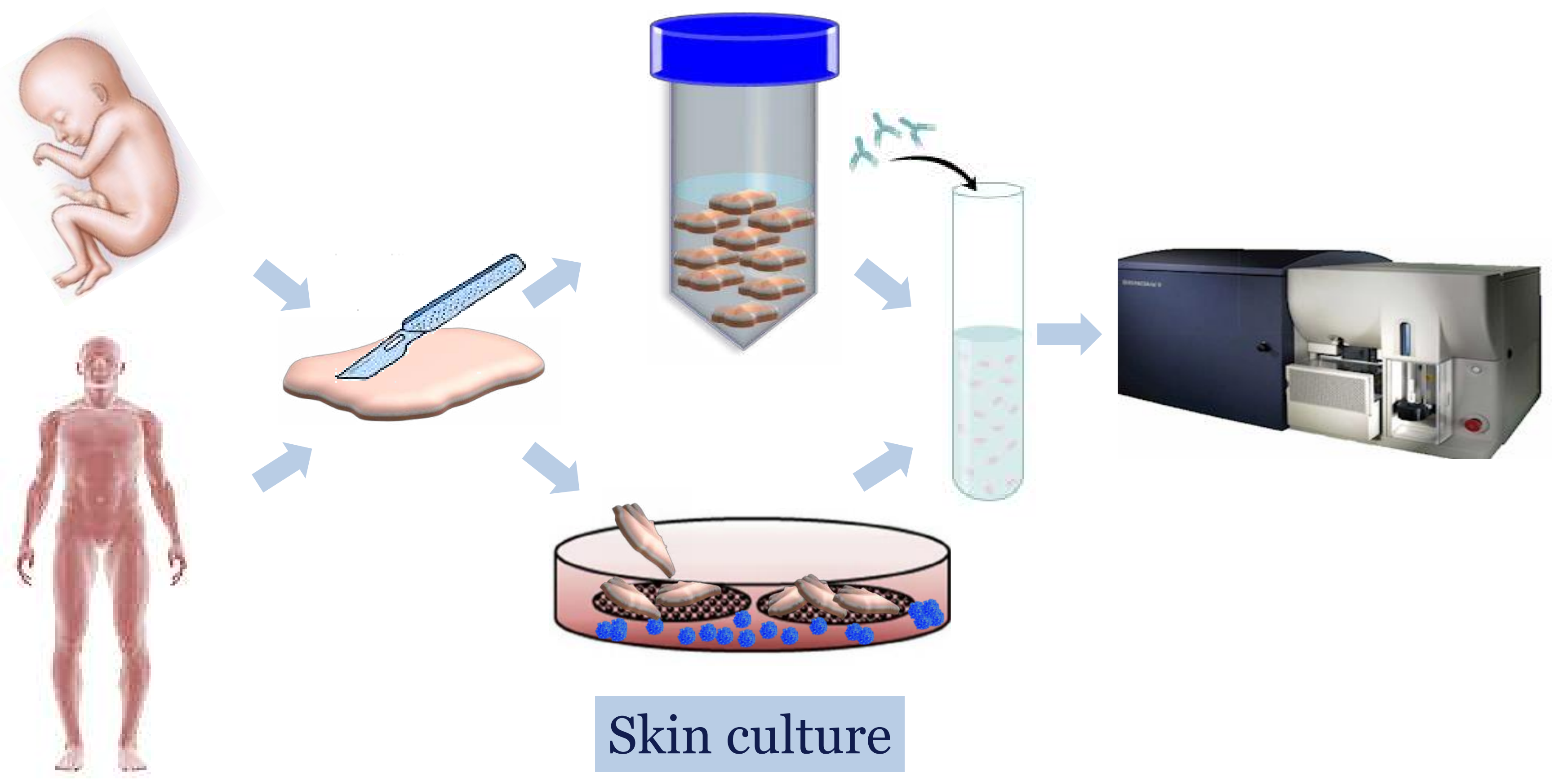

Figure 1. Schematic diagram of skin T cell isolation. Skin biopsies from adults (age range: 30 50 years) and prenatal skin (age range: 18-22 weeks EGA,) are taken. Skin is either minced and used for enzymatic digestion or loaded on tantalum pre-coated grids allowing $T$ cells to migrate Out of the skin. Flow cytometry is used for T cell subset analysis.

Results
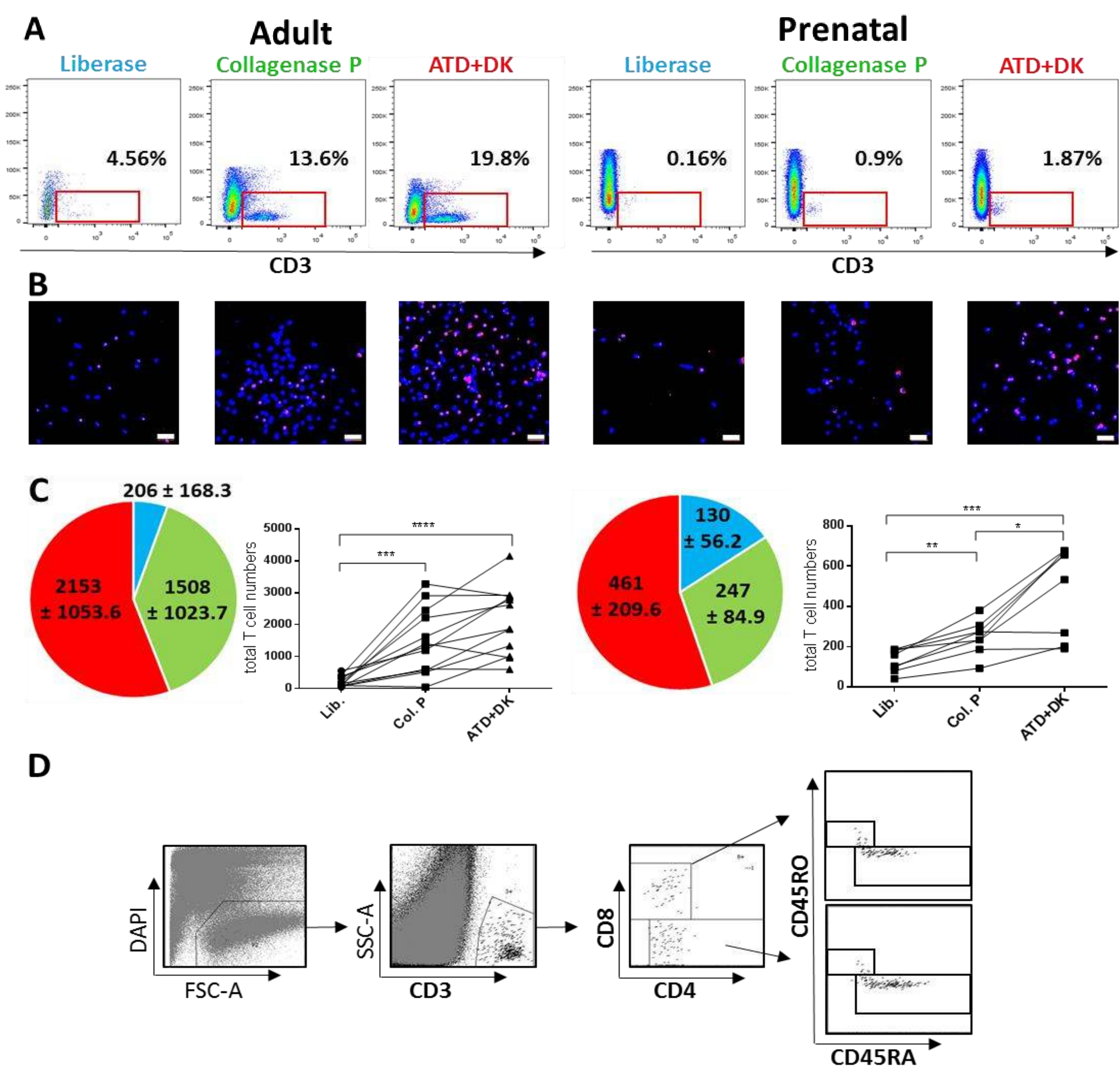

Figure 2: Comparative analysis of T cell isolation procedures from adult and prenatal skin. (A) Dot blots demonstrating CD3 expression upon indicated isolation procedures from adult $(n=12)$ and prenatal $(n=8)$. ATD: automatic tissue dissociator, DK: dissociation kit. (B) Confocal microscopy analysis of isolated skin cells stained for CD3 (red) and DAPI (blue). Scale bar: $50 \mu \mathrm{m}$. (C) Pie charts showing frequency of live CD 3 T cells from adult and prenatal skin using liberase/dispase (blue) collagenase $P$ (green) and ATD+DK (red). Graphs showing statistical analyses of CD3+ $T$ cell frequency upon denoted isolation procedures. Student's $t$ test. "P $\leq 0.05$, $P \leq 0.01$, ***P $P \leq 0.005$ $* * * * \times \leq 0.0001$. (D) Representative flow cytometry scatter plots of the sorting panels for freshly

\section{Conclusion}

These studies expand our understanding of the formation of important prerequisites for a functional skin immune system and consequently help to better understand many human skin diseases characterized by T cell activation and proliferation.
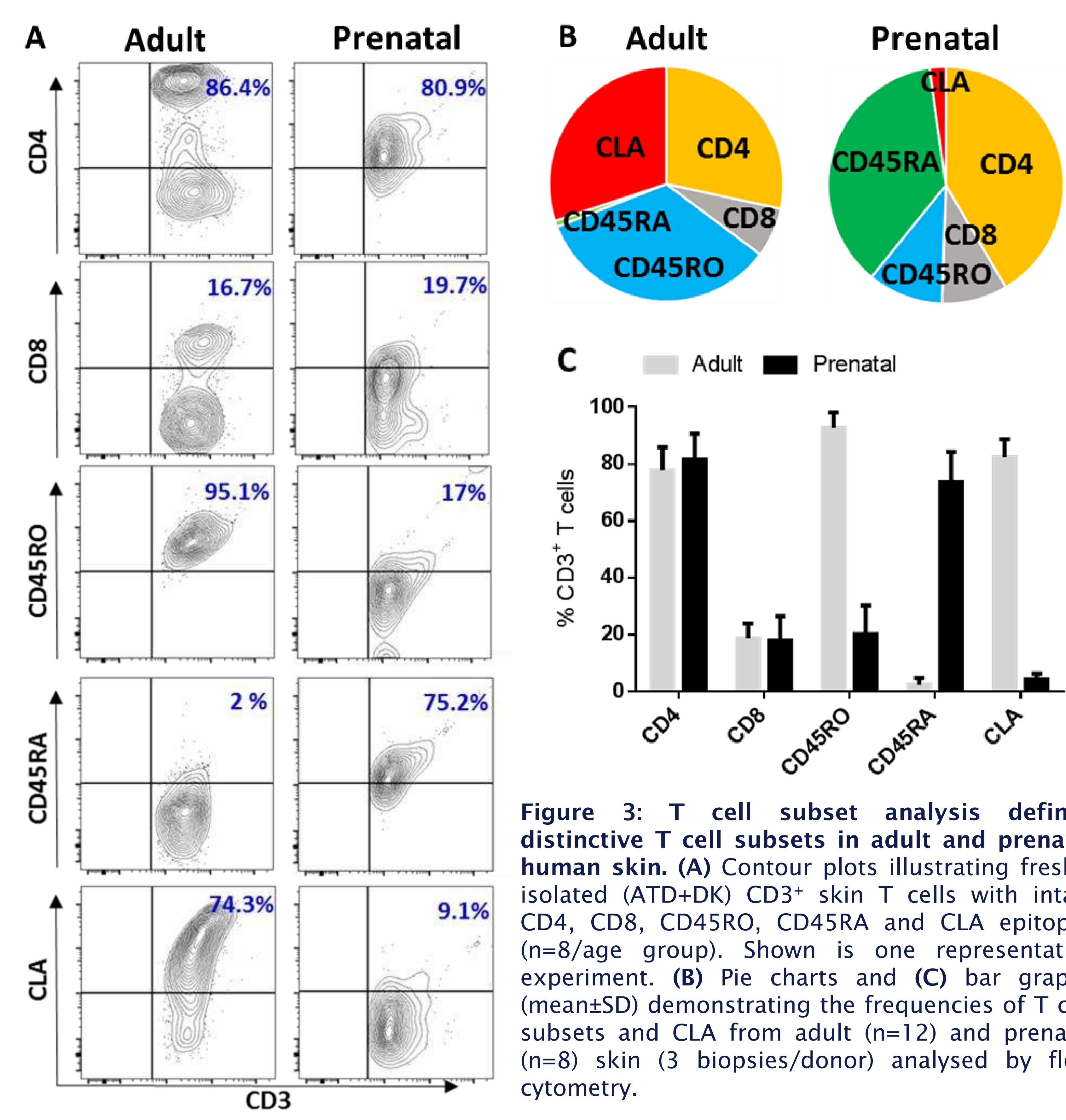

Figure 3: $T$ cell subset analysis defines distinctive $T$ cell subsets in adult and prenatal human skin. (A) Contour plots illustrating freshly CD4, CD8, CD45RO CD45RA and CLA epitapes (n=8/age, Group). Shown is one representative experiment. (B) Pie charts and (C) bar graphs (mean+SD) demonstrating the frequencies of $T$ cell subsets and CLA from adult $(n=12)$ and prenatal $(n=8)$ skin (3 biopsies/donor) analysed by flow

A<smiles>[GeH3]</smiles>
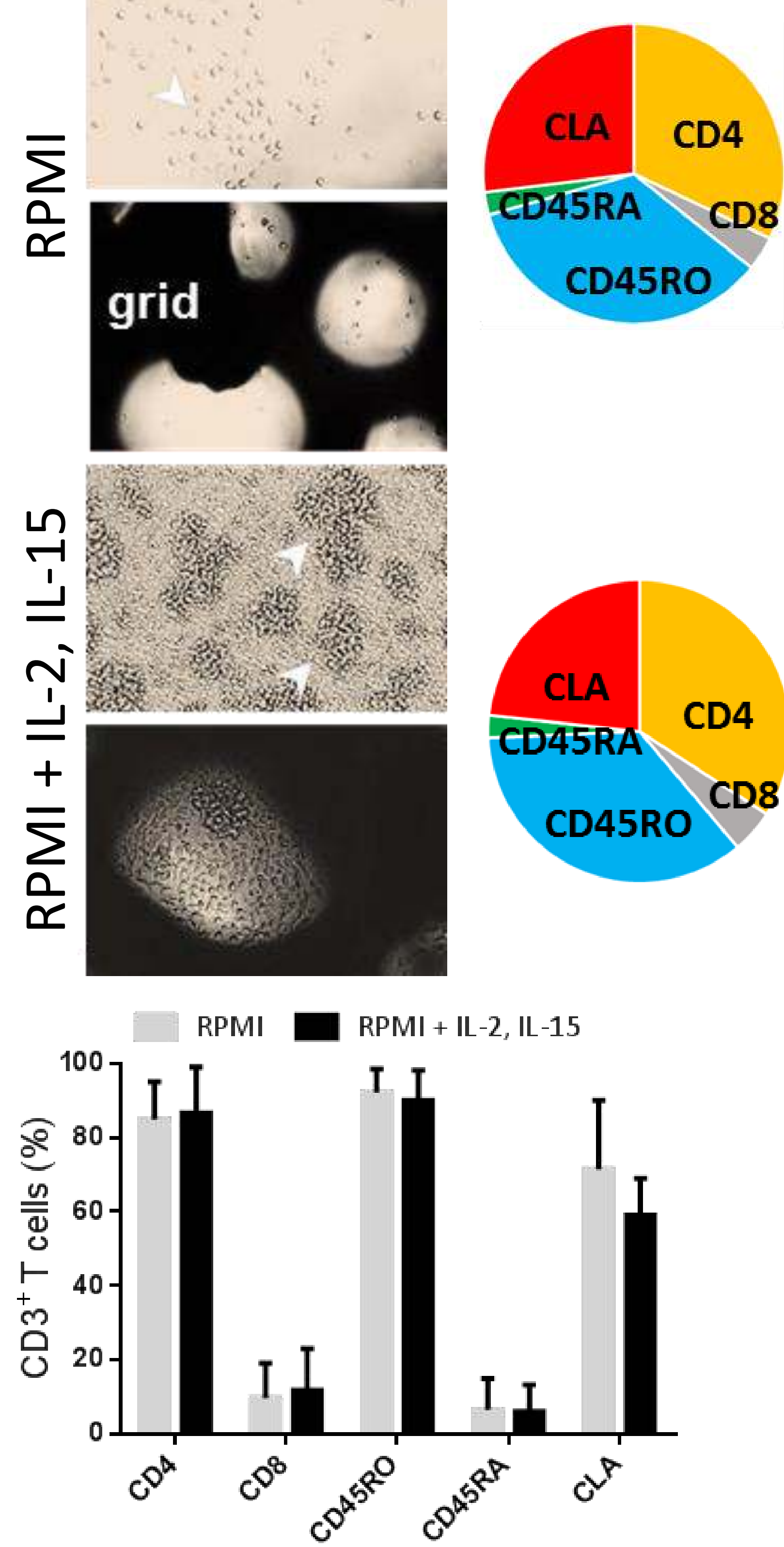

B
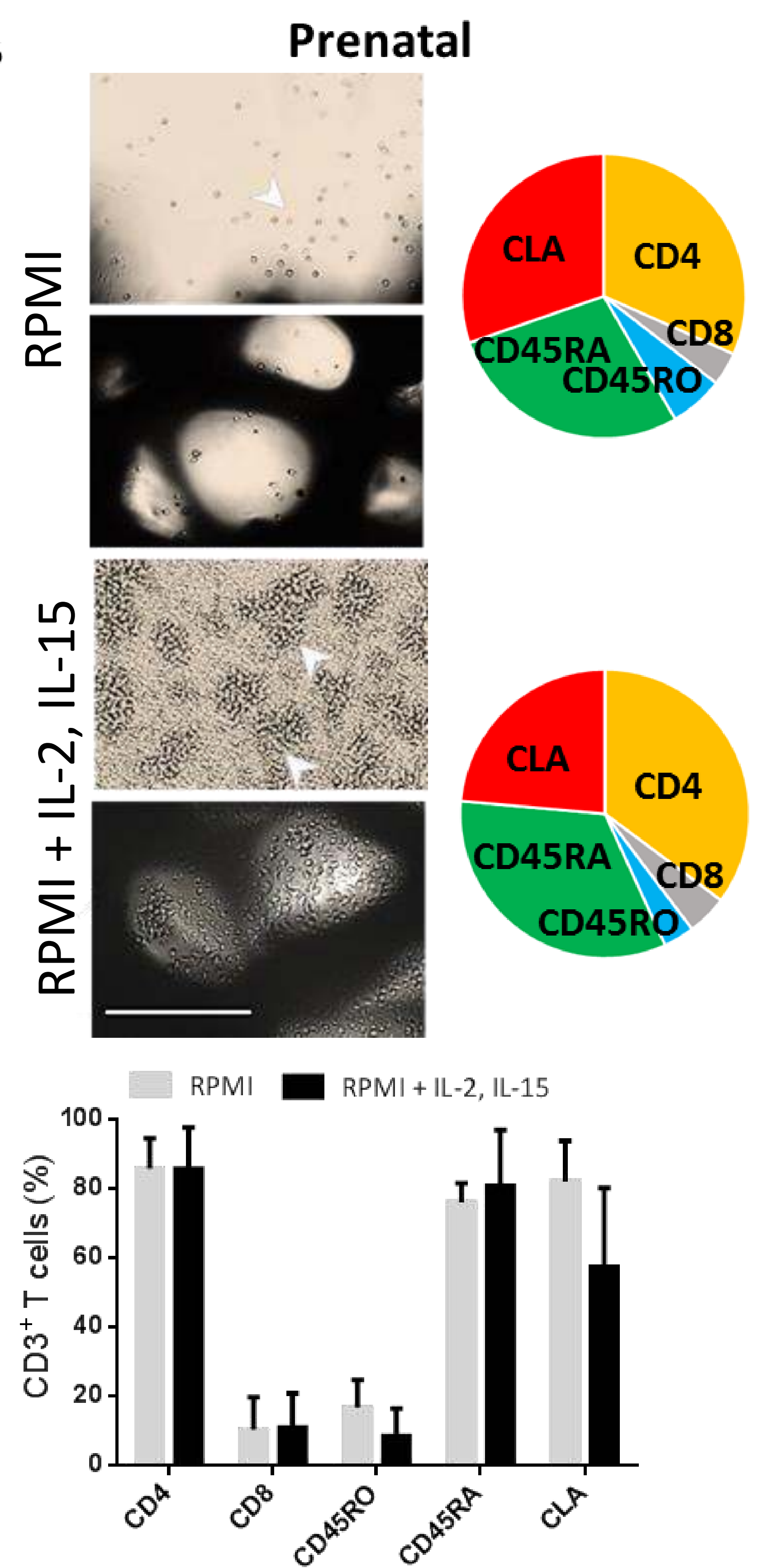

Figure 4: Analysis of expanded T cells. Biopsies from adult and prenatal skin were cultured on

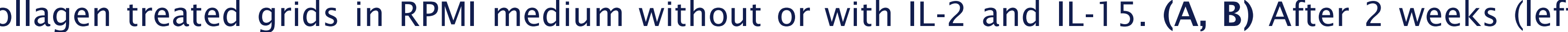
panel) single $T$ cells are visible in RPMI medium alone (arrowhead), while huge proliferating Custers, ifrespective if adult or prenatal skin was used, are detectable in cultures containing IL-2

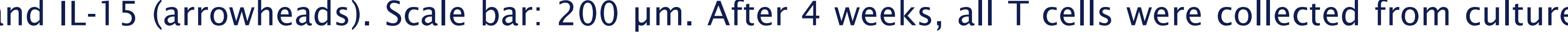
wells, stained with indicated surface markers and analyzed by flow cytometry. Pie charts show (C, D) Burnof (C, D) Bar graphs show percentages of expanded CD3+ $T$ cells from adult $(n=9)$ and prenatal $(n=8)$ 位

Adult

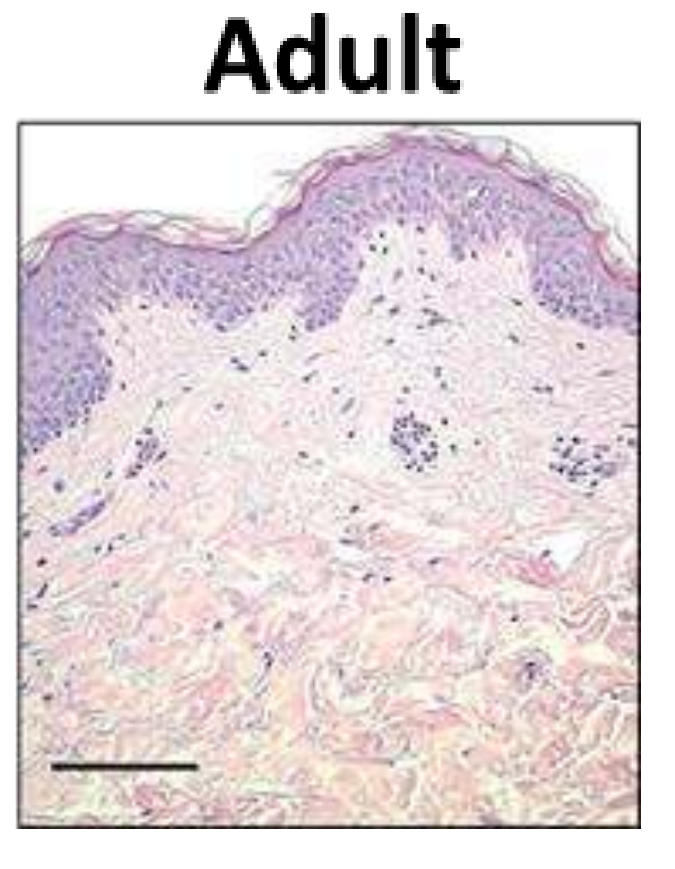

Prenatal

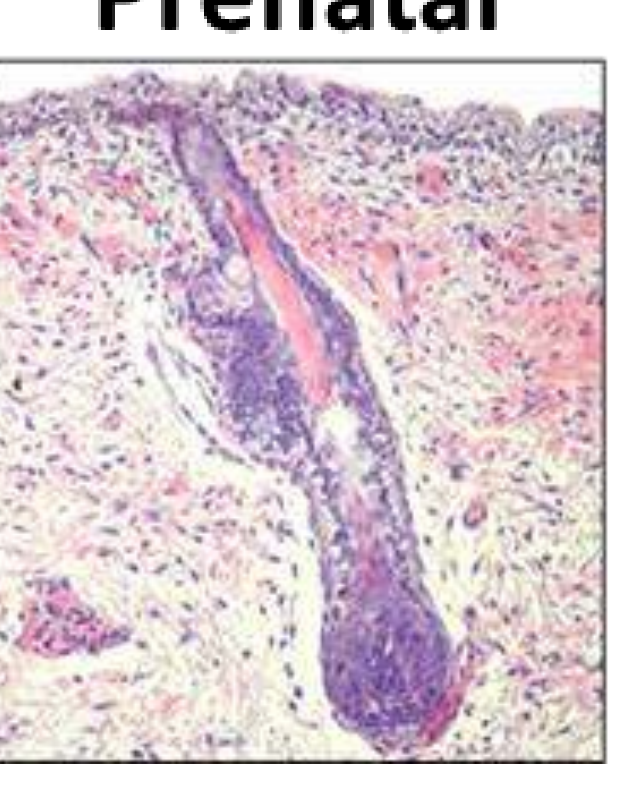

Figure 5: Histological examination of H\&E-stained skin paraffin sections showing the morphology of fetal and adult skin. Hair follicles are detectable in fetal skin at 20 week of EGA. The four layers of the epidermis stratum corneum, granulosum, spinosum and basale are visible in the adult skin but cannot be distinguished yet in prenatal skin. Scale bar $=$ $00 \mu \mathrm{m}$. 